\title{
A Markov Switching Re-evaluation of Event-Study Methodology
}

\author{
Rosella Castellano ${ }^{1}$ and Luisa Scaccia ${ }^{2}$ \\ 1 Dip. di Istituzioni Economiche e Finanziarie, Università di Macerata \\ via Crescimbeni 20,62100 Macerata, Italy, castellano@unimc.it \\ 2 Dip. di Istituzioni Economiche e Finanziarie, Università di Macerata \\ via Crescimbeni 20, 62100 Macerata, Italy, scaccia@unimc.it
}

\begin{abstract}
This paper reconsiders event-study methodology in light of evidences showing that Cumulative Abnormal Return (CAR) can result in misleading inferences about financial market efficiency and pre(post)-event behavior. In particular, CAR can be biased downward, due to the increased volatility on the event day and within event windows. We propose the use of Markov Switching Models to capture the effect of an event on security prices. The proposed methodology is applied to a set of 45 historical series on Credit Default Swap (CDS) quotes subject to multiple credit events, such as reviews for downgrading. Since CDSs provide insurance against the default of a particular company or sovereign entity, this study checks if market anticipates reviews for downgrading and evaluates the time period the announcements lag behind the market.
\end{abstract}

Keywords: hierarchical Bayes, Markov switching models, credit default swaps, event-study

\section{Introduction}

An event-study is the name given to the empirical investigation of the relationship between security prices and economic events. It allows to estimate and draw inferences about the impact of an event in a particular period or over several periods. The most common approach involves four steps: 1) identification of the event dates for a sample of securities subject to the disclosure item of interest (i.e. rating announcements) and creation of equally sized event windows around each event date; 2) selection of an appropriate reference period preceding each event window (the so-called estimation period), used to estimate the mean and standard deviation of the returns of each security, under normal market conditions; 3) computation of Abnormal Returns (ARs) on each security supposed to be influenced by the event and for each event window around the announcement date; 4) computation of the mean ARs across securities in the sample, possibly cumulated over the event windows, and comparison with the mean returns estimated under normal market conditions, through parametric and non parametric test statistics (Brown and Warner (1985); Kothari and Warner (1997)). 
In most situations, event-study tests relying on the Cumulated Abnormal Return (CAR) methodology may provide misleading results because of the kurtosis and volatility clustering characterizing financial time series. Therefore, we propose to incorporate into the classical event-study methodology the ability of Markov Switching Models, also known as Hidden Markov Models (HMMs), to model state-dependent means and variances of the ARs. In practice, instead of performing the hypothesis testing described in the fourth step of the above illustrated methodology, we model the ARs in each event window through an HMM characterized by two states, normal and abnormal market conditions, and look at the probability that the generating process is in each of the two states, at any time in the event window. In this way, we explicitly account for the kurtosis and the volatility clustering commonly observed in financial time series. We adopt a Bayesian perspective and rely on the flexibility of hierarchical modeling. It is worth to notice that HMMs have been successfully applied to financial time series. For instance, segmented time-trends in the US dollar exchange rates (Engel and Hamilton (1990), Castellano and Scaccia (2010), Otranto and Gallo (2002)), stylized facts about daily returns (Rydén et al. (1998)), option prices and stochastic volatilities (Rossi and Gallo (2006)), temporal behavior of daily volatility on commodity returns (Haldrup and Nielsen (2006)) have been modeled via HMMs.

In this paper we apply the proposed methodology to study the reactions of Credit Default Swap (CDS) quotes to reviews for downgrading announced by three major credit rating agencies (Moody's, Fitch and Standard\&Poor's), in order to examine if and to what extent this market responds to these announcements which should reflect the latest available information. The focal idea is to analyze if rating agencies have access to non-public information, implying that their announcements can be viewed as conveying extra information to the market, and if the size and variance of ARs may provide information about the creditworthiness of a specific company. Basically, if reviews for downgrading convey new information to the market, CDS quotes should react after the announcement and a significant increase in market volatility should be expected at the event day or after the announcements. Otherwise, it might be possible that reviews for downgrading only reflect information already discounted by the market, implying that CDS quotes do not react to watchlisting and abnormal performances are observed before the announcements.

Data over the period 2004 - 2009 for 45 international companies belonging to different credit grades are taken into consideration and the effects of reviews for downgrading on CDS quote generating process are investigated.

The paper proceeds as follows: the Markov Switching re-evaluation of the classical event-study methodology and the priors on model parameters are illustrated in Section 7, Section 3 deals with computational implementation; 
Section 4 discusses the application on CDS quotes; conclusions are reported in Section 5 .

\section{The revised event-study methodology}

The approach proposed in this paper to investigate the above mentioned hypotheses is based on the Markov Switching re-evaluation of event-study methodology. In this context, the event window is set equal to almost three months, starting 60 business days before a review for downgrading and ending 20 business days after the announcement, thus the series considered will be indexed by $t \in[-60,+20]$. In the following, we will use $t_{0}$ and $T$ to indicate, respectively the starting day and the ending day of the event window, so that $t_{0}=-60$ and $T=20$. If the announcement is fully anticipated, then the CDS quote generating process should adjust prior to $t=0$, the day of the announcement. If the rating announcement has a new informational content, it should have an effect on price at $t=0$ and, in the case of postannouncement effect, the impact of the review for downgrading might be delayed after $t=0$. This choice of the event window aims at analyzing the reactions of the markets before and around the day of the announcement, the period in which a potential market reaction is expected, as many studies have shown (Kliger and Sarig (2000); Steiner and Heinke (2001); Norden and Weber (2004); Hull et al. (2004); Heinke (2006)).

To examine the impact of reviews for downgrading on the generating process of CDS quotes, we focus on the daily returns of each CDS, defined as:

$$
R_{t}=P_{t}-P_{t-1}
$$

where $P_{t}$ is the market value of the CDS at time $t$. Daily returns are then used to calculate standardized ARs as:

$$
y_{t}=\frac{R_{t}-\bar{R}}{s_{R}},
$$

where the sample mean and standard deviation of the returns for each CDS subject to review for downgrading are estimated over an estimation period of 100-day preceding each event window, so that $\bar{R}=\sum_{t=-160}^{-61} R_{t} / 100$ and $s_{R}^{2}=\sum_{t=-160}^{-61}\left(R_{t}-\bar{R}\right)^{2} / 99$.

In a HMM formulation, the distribution of each standardized AR, $y_{t}$, is assumed to depend on an unobserved (hidden) variable, denoted by $s_{t}$, that takes on values from 1 to $k$. The vector of hidden variables $s=\left(s_{t}\right)_{t=t_{0}}^{T}$ characterizes the "state" or "regime" in which the AR generating process is at any time $t$. The $y_{t}$ are assumed to be independent, conditional on the $s_{t}$ 's:

$$
y_{t} \mid s_{t} \sim f_{s_{t}}\left(y_{t}\right) \quad \text { for } t=t_{0}, t_{0}+1, \ldots, T,
$$

with $f_{s_{t}}(\cdot)$ being a specified density function. We further postulate that the dynamics of $s$ are described by a Markov Chain with transition matrix $\boldsymbol{\Lambda}=$ 
$\left(\lambda_{i j}\right)_{i, j=1}^{k}$, implying that $s_{t}$ is presumed to depend on the past realizations of $\boldsymbol{y}$ and $\boldsymbol{s}$, only through $s_{t-1}$ :

$$
p\left(s_{t}=j \mid s_{t-1}=i\right)=\lambda_{i j} .
$$

Since we apply the HMM to ARs of CDSs, we implicitly assume $y_{t}$ to be normally distributed, so that the model in (1) becomes

$$
y_{t} \mid \boldsymbol{s}, \boldsymbol{\mu}, \boldsymbol{\sigma} \sim \phi\left(\cdot ; \mu_{s_{t}}, \sigma_{s_{t}}^{2}\right)
$$

conditional on means $\boldsymbol{\mu}=\left(\mu_{i}\right)_{i=1}^{k}$ and standard deviations $\boldsymbol{\sigma}=\left(\sigma_{i}\right)_{i=1}^{k}$, where $\phi\left(\cdot ; \mu_{i}, \sigma_{i}^{2}\right)$ is the density of the $N\left(\mu_{i}, \sigma_{i}^{2}\right)$. Thus, if $s_{t}=i, y_{t}$ is assumed to be drawn from a $N\left(\mu_{i}, \sigma_{i}^{2}\right)$. Notice that, if we let $\boldsymbol{\pi}$ being the stationary vector of the transition matrix, so that $\boldsymbol{\pi}^{\prime} \boldsymbol{\Lambda}=\boldsymbol{\pi}^{\prime}$, and we integrate out $s_{t}$ in (2) using its stationary distribution, the model in (2) can be analogously formalized as

$$
y_{t} \mid \boldsymbol{\pi}, \boldsymbol{\mu}, \boldsymbol{\sigma} \sim \sum_{i=1}^{k} \pi_{i} \phi\left(\cdot ; \mu_{i}, \sigma_{i}^{2}\right) \quad \text { for } \quad t=t_{0}, t_{0}+1, \ldots, T .
$$

In this paper the number of states, $k$, is assumed equal to 2 , as briefly explained in Section 4 In a forthcoming paper, $k$ will be considered unknown and subject to inference, as well as the other parameters of the model.

In a Bayesian context, the uncertainty on the parameters of the model is formalized using appropriate prior distributions. Weakly informative priors are chosen, by introducing an hyperprior structure. We assume:

a) $\mu_{i} \mid \sigma_{i}^{2} \sim \mathcal{N}\left(\xi, \kappa \sigma_{i}^{2}\right)$, independently for each $i=1, \ldots, k$.

b) $\sigma_{i}^{-2} \sim \mathcal{G}(\eta, \zeta)$, independently for each $i=1, \ldots, k$, with the mean and the variance of the Gamma distribution being $\eta / \zeta$ and $\eta / \zeta^{2}$.

c) $\kappa \sim \mathcal{I G}(q, r)$, with $\mathcal{I G}$ denoting the Inverse Gamma distribution.

d) $\zeta \sim \mathcal{G}(f, h)$.

e) $\lambda_{i j} \sim \mathcal{D}\left(\boldsymbol{\delta}_{j}\right)$, for $i=1, \ldots, k$ where $\mathcal{D}$ denotes the Dirichlet distribution and $\boldsymbol{\delta}_{j}=\left(\delta_{i j}\right)_{i=1}^{k}$.

\section{Computational implementation}

In order to approximate the posterior joint distribution of all the parameters of the above HMM, we apply Markov Chain Monte Carlo (MCMC) methods and exploit the natural conditional independence structure of the model so that the joint distribution of all variables, conditional to the fixed values of the hyperparameters, is:

$$
\begin{aligned}
& p(\boldsymbol{y}, \boldsymbol{\mu}, \boldsymbol{\sigma}, \boldsymbol{s}, \boldsymbol{\Lambda}, \zeta, \kappa \mid \boldsymbol{\delta}, f, h, q, r, \xi, \eta, k) \\
& \quad=p(\boldsymbol{y} \mid \boldsymbol{s}, \boldsymbol{\mu}, \boldsymbol{\sigma}) p(\boldsymbol{s} \mid \boldsymbol{\Lambda}) p(\boldsymbol{\Lambda} \mid \boldsymbol{\delta}, k) p(\boldsymbol{\mu} \mid \boldsymbol{\sigma}, \xi, \kappa, k) p(\kappa \mid r, q) p(\boldsymbol{\sigma} \mid \eta, \zeta, k) p(\zeta \mid f, h) .
\end{aligned}
$$

To generate realizations from the posterior joint distribution, the parameters of the model are in turn updated, by means of Gibbs sampler. At each sweep of the MCMC algorithm, the following steps are performed: 
Updating $\boldsymbol{\Lambda}$. The $i$-th row of $\boldsymbol{\Lambda}$ is sampled from $D\left(\delta_{i 1}+n_{i 1}, \ldots, \delta_{i k}+n_{i k}\right)$, where $n_{i j}=\sum_{t=t_{0}}^{T-1} I\left\{s_{t}=i, s_{t+1}=j\right\}$ is the number of transitions from regime $i$ to regime $j$ and $I\{\cdot\}$ denotes the indicator function.

Updating $s$. The standard solution for updating $s$ would be to sample $s_{t_{0}}, \ldots, s_{T}$ one at a time from $t=t_{0}$ to $t=T$, drawing values from their full conditional distribution $p\left(s_{t}=i \mid \cdots\right) \propto \lambda_{s_{t-1} i} \phi\left(y_{t} ; \mu_{i}, \sigma_{i}^{2}\right) \lambda_{i s_{t+1}}$ where '...' denotes 'all other variables'. For a faster mixing algorithm (Scott (2002)), we instead sample $\boldsymbol{s}$ from $p(\boldsymbol{s} \mid \boldsymbol{y}, \boldsymbol{\Lambda})$ through a stochastic version of the forward-backward recursion. The forward recursion produces matrices $\boldsymbol{P}_{t_{0}+1}, \boldsymbol{P}_{t_{0}+2}, \ldots, \boldsymbol{P}_{T}$, where $\boldsymbol{P}_{t}=\left(p_{t i j}\right)$ and $p_{t i j}=$ $p\left(s_{t-1}=i, s_{t}=j \mid y_{1}, \ldots, y_{t}, \boldsymbol{\Lambda}\right)$. In words, $\boldsymbol{P}_{t}$ is the joint distribution of $\left(s_{t-1}=i, s_{t}=j\right)$ given parameters and observed data up to time $t . \boldsymbol{P}_{t}$ is computed from $\boldsymbol{P}_{t-1}$ as $p_{t i j} \propto p\left(s_{t-1}=i, s_{t}=j, y_{t} \mid y_{1}, \ldots, y_{t-1}, \boldsymbol{\Lambda}\right)=$ $p\left(s_{t-1}=i \mid y_{1}, \ldots, y_{t-1}, \boldsymbol{\Lambda}\right) \lambda_{i j} \phi\left(y_{t} ; \mu_{j}, \sigma_{j}^{2}\right)$ with proportionality reconciled by $\sum_{i} \sum_{j} p_{t i j}=1$, where $p\left(s_{t-1}=i \mid y_{1}, \ldots, y_{t-1}, \boldsymbol{\Lambda}\right)=\sum_{j} p_{t-1, i, j}$ can be computed once $\boldsymbol{P}_{t-1}$ is known. The recursion starts computing $p\left(s_{t_{0}}=\right.$ $\left.i \mid y_{t_{0}}, \boldsymbol{\Lambda}\right) \propto \phi\left(y_{t_{0}} ; \mu_{i}, \sigma_{i}^{2}\right) \pi_{i}$ and thus $\boldsymbol{P}_{t_{0}+1}$. The stochastic backward recursion begins by drawing $s_{T}$ from $p\left(s_{T} \mid \boldsymbol{y}, \boldsymbol{\Lambda}\right)$, then recursively drawing $s_{t}$ from the distribution proportional to column $s_{t+1}$ of $\boldsymbol{P}_{t+1}$. In this way, the stochastic backward recursion samples from $p(\boldsymbol{s} \mid \boldsymbol{y}, \boldsymbol{\Lambda})$, factorizing this as $p(\boldsymbol{s} \mid \boldsymbol{y}, \boldsymbol{\Lambda})=p\left(s_{T} \mid \boldsymbol{y}, \boldsymbol{\Lambda}\right) \prod_{t=t_{0}}^{T-1} p\left(s_{T-t} \mid s_{T}, \ldots, s_{T-t+1}, \boldsymbol{y}, \boldsymbol{\Lambda}\right)$ where $p\left(s_{T-t}=i \mid s_{T}, \ldots, s_{T-t+1}, \boldsymbol{y}, \boldsymbol{\Lambda}\right)=p\left(s_{T-t}=i \mid s_{T-t+1}, y_{t_{0}}, \ldots, y_{T-t+1}, \boldsymbol{\Lambda}\right)$ $\propto p_{T-t+1, i, s_{T-t+1}}$.

Updating $\mu$. Letting $n_{i}$ being the number of observations currently allocated in regime $i$, the $\mu_{i}$ can be updated by drawing them independently from $\mu_{i} \mid \cdots \sim \mathcal{N}\left(\frac{\kappa \sum_{t: s_{t}=i} y_{t}+\xi}{1+\kappa n_{i}}, \frac{\sigma_{i}^{2} \kappa}{1+\kappa n_{i}}\right)$.

Updating $\kappa$. We sample $\kappa^{-1}$ from $\kappa^{-1} \mid \cdots \sim \mathcal{G}\left(q+\frac{k}{2}, r+\frac{1}{2} \sum_{i=1}^{k} \frac{\left(\mu_{i}-\xi\right)^{2}}{\sigma_{i}^{2}}\right)$. Updating $\sigma$. For identifiability purpose, we adopt a unique labeling in which the $\sigma_{i}$ 's are in increasing numerical order. Hence, their joint prior distribution is $k$ ! times the product of the individual $\mathcal{I} \mathcal{G}$ densities, restricted to the set $\sigma_{1}<\sigma_{2}<\ldots<\sigma_{k}$. The $\sigma_{i}$ can be drawn independently from $\sigma_{i}^{-2} \mid \cdots \sim \mathcal{G}\left(\eta+\frac{1}{2}\left(n_{i}+1\right), \zeta+\frac{1}{2} \sum_{t: s_{t}=i}\left(y_{t}-\mu_{i}\right)^{2}+\frac{1}{2 \kappa}\left(\mu_{i}-\xi\right)^{2}\right)$. The move is accepted, provided the invariance of the order.

Updating $\zeta$. We sample $\zeta$ from $\zeta \mid \cdots \sim \mathcal{G}\left(f+k \eta, h+\sum_{i=1}^{k} \sigma_{i}^{-2}\right)$.

\section{An Application}

To estimate the parameters of the model and the posterior probabilities of $y_{t}$ being in each of the states, at any time $t \in[-60,+20]$, we performed 100,000 sweeps of the MCMC algorithm, allowing for a burn-in of 10,000 sweeps. Notice that our data set considers 45 historical series of CDSs and 
related reviews for downgrading, leading to 57 non-overlapping events (in the sense that we only analyze subsequent event time windows including one event) and, thus, to 57 different ARs series. The MCMC algorithm was run independently for each of the 57 series of ARs. Since most of the ARs in the events windows are well represented by two regimes, characterized by high and low volatility respectively, we set $k=2$.

Performing a preliminary analysis, we find that the probability of being in the high volatility state in each event window of the data base, is characterized by different patterns. As a consequence, cluster analysis is performed by applying the k-means algorithm to the event windows. Using the Schwarz criterion, five clusters were selected.

Figure 1 shows the averaged posterior probabilities of being in the high volatility regime for the event windows belonging to the first four clusters. Cluster (a) highlights that reviews for downgrading are fully anticipated by the market. In this cluster the probability of being in the high volatility regime is larger than 0.5 almost fifty days before the announcement occurs and it is relevant the absence of announcement and post-announcement effects. This cluster is the most interesting among those under consideration, because it shows that reviews for downgrading announced by rating agencies, which should reflect the latest available information, are largely anticipated by an increase in the volatility of ARs and announcements do not convey new information to the market.

Cluster (b) groups event windows for which the volatility of the abnormal returns is drawn from the high volatility state almost twenty days before the event occurrence. This implies that CDS market anticipates the announcements. At the same time, the ARs of the event windows belonging to this cluster show that volatility remains in the high state also around $t=0$ and for twenty days after the announcements, highlighting also the presence of announcement and post-announcement effects. From a financial point of view, it may be useful to note that this behavior is probably due to the specific nature of the events under consideration, which represent only reviews for possible downgrades and not effective downgrades, meaning that announcements are not always followed by a reduction in the effective creditworthiness of the reference entity. Persistence of the high volatility state after $t=0$ may be interpreted as a measure of the market's expectation regarding the future effective downgrading (in the data base under consideration the $65 \%$ of reviews for downgrading is followed by effective downgrading in an average period of 80 days).

The ARs of the event windows belonging to cluster (c) show that market volatility anticipates the reviews for downgrading by almost ten days and decreases very quickly about ten days after the event. For the ARs of the event windows belonging to this cluster, we can conclude that nevertheless the rating announcement is anticipated by the market via an increase in volatility, it is still present an announcement effect, while the post announcement effect 

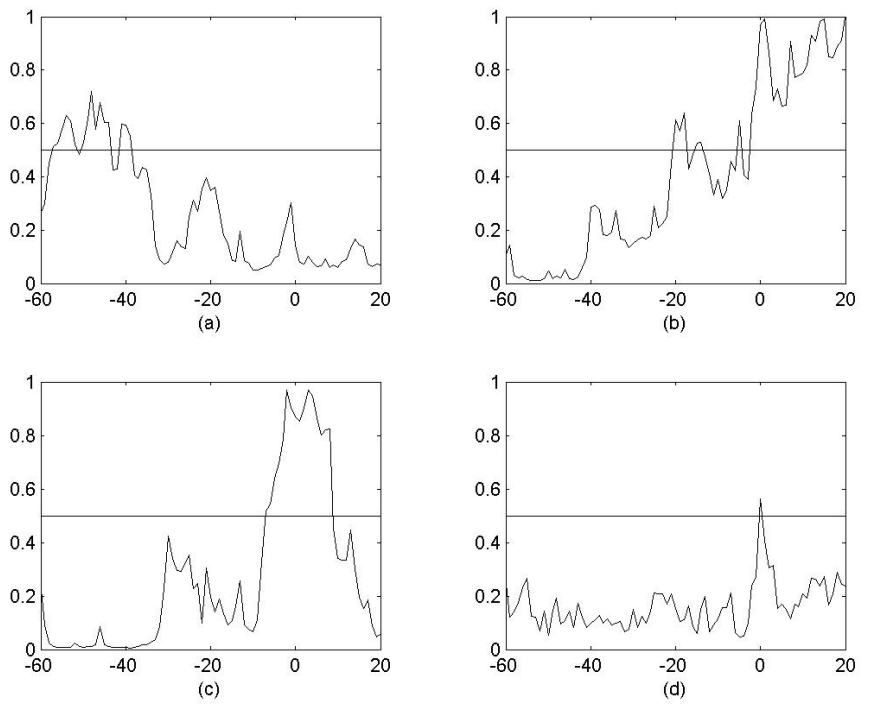

Fig. 1. Estimated mean posterior probabilities of being in the high volatility regime for series belonging to four different clusters.

is largely reduced. The fourth cluster (d) collects event windows for which the mean posterior probability of being in a high volatility state exceeds 0.5 only at $t=0$, implying the existence of an announcement effect.

The fifth cluster, not shown here, is a residual cluster which collects event windows characterized by a higher degree of heterogeneity than those in the other clusters. In this case, the mean posterior probability of being in a high volatility regime is not particularly meaningful.

\section{Conclusions}

This study was conducted to answer fundamental questions about the effectiveness of event-study methodology in capturing the effect of events on financial asset behavior. We used a data set on CDS quotes and credit rating data. It covers the period from June, 2004 to October, 2009. We choose the five-year CDS quotes since this is the benchmark maturity in the CDS market. We collected the reviews for downgrading (watchlistings) announced by three major rating agencies (Fitch, Moody's and Standard\&Poor's) for the sample firms in order to verify whether announcements carry new information to the CDS market or not. At the end of the sampling period, we selected 57 non-overlapping events.

Since the estimated effect of events on security behavior investigated by classic event-study methodology may be biased downward, because of the 
averaging effect and the increased volatility around events, we re-evaluate the classical approach by introducing a HMM characterized by two volatility regimes (high and low) to model the ARs of each event window. We find that CDS market anticipates reviews for downgrading and we show, through cluster analysis, that the anticipation period can follows different patterns.

\section{References}

BROWN, S. and WARNER, J. (1985): Using daily stock returns: The case of event studies. Journal of Financial Economics 14 (1), 3-31.

CASTELLANO, R. and SCACCIA, L. (2010): Bayesian hidden Markov models for financial data. In: F. Palumbo, C.N. Lauro and M.J. Greenacre (Eds.): Data Analysis and Classification: From Exploration to Confirmation. Springer, Berlin-Heidelberg, 453-461.

ENGEL, C. and HAMILTON, J.D. (1990): Long swings in the dollar: Are they in the data and do markets know it? The American Economic Review 80 (4), 689-713.

HALDRUP, N. and NIELSEN, M.O. (2006): A regime switching long memory model for electricity prices. Journal of econometrics 135 (1-2), 349-376

HEINKE, V.G. (2006): Credit spread volatility, bond ratings and the risk reduction effect of watchlistings. International Journal of Finance and Economics 11 (4), 293-303.

HULL, J., PREDESCU, M. and WHITE, A. (2004): The relationship between credit default swap spreads, bond yields, and credit rating announcements. Journal of Banking and Finance 28 (11), 2789-2811.

KLIGER, D. and Sarig, O. (2000): The information value of bond ratings. The Journal of Finance 55 (6), 2879-2902.

KOTHARI, S. and WARNER, J. (1997): Measuring long-horizon security price performance. Journal of Financial Economics 43 (3), 301-339.

NORDEN, L. and WEBER, M. (2004): Informational efficiency of credit default swap and stock markets: The impact of credit rating announcements. Journal of Banking and Finance 28 (11), 2813-2843.

OTRANTO, E. and GALLO, G.(2002): A nonparametric Bayesian approach to detect the number of regimes in Markov switching models. Econometric Reviews 21 (4), $477-496$.

ROSSI, A. and GALLO, G. (2006): Volatility estimation via hidden Markov models. Journal of Empirical Finance 13 (2), 203-230.

RYDÉN, T., TERÄSVIRTA, T. and rASBRINK, S. (1998): Stylized facts of daily return series and the hidden Markov model. Journal of Applied Econometrics 13 (3), 217-244.

STEINER, M. and HEINKE, V.G. (2001): Event study concerning international bond price effects of credit rating actions. International Journal of Finance and Economics 6 (2), 139-15\%. 